1997 will facilitate future research on the etiology of CMs. (Hobbs CA, Cleves MA, Simmons CJ. Genetic epidemiology and congenital malformations: From the chromosome to the crib. Arch Pediatr Adolesc Med April 2002;156:315-320). (Reprints: CM Hobbs MD, PhD, Dept Pediatrics, University of Arkansas, 11219 Financial Ctr Parkway, Ste 250, Little Rock, AR 72211).

COMMENT. The identification of genes and environmental factors involved in the etiology of congenital malformations will aid in the prevention, diagnosis, prognosis, and treatment of congenital malformations.

A Neuropathological Approach to the classification and cause of genetic defects and specifically holoprosencephaly is provided by Sarnat HB and FloresSarnat L at Cedars- Sinai Medical Center, Los Angeles, CA (I Child Neurology 2001;16:918-931). The need for a new classification for holoprosencephaly is suggested that integrates morphological and genetic criteria. Genes expressed in the neural tube may have rostrocaudal and mediolateral gradients in axes other than the vertical. A rostrocaudal gradient extending to the mesencephalic neuromere may affect the development of the bones of the face and explain the midfacial hypoplasia seen with holoprosencephaly. Four defective genes have been identified, three having a ventrodorsal gradient of expression (SHH, SIX3, and TGIF) and one a dorsoventral gradient (ZIC2).

Sarnat $\mathrm{HB}$ and associates also report 2 infant cases of agenesis of the mesencephalon and metencephalon with certebellar hypoplasia, a rare congenital malformation resulting in early death from impaired central respiratory drive (Pediatric and Developmental Pathology 2002;5:54-68). The authors speculate that the defect results from a mutation or deletion in the EN2 gene.

\title{
CHIARI TYPE I MALFORMATION AND SEIZURES
}

Four children with epilepsy, ranging in age from 8 to 15 years, and diagnosed with Chiari type I malformation by brain magnetic resonance imaging (MRI), are reported from La Sapienza University, Rome, Italy. Seizures were complex partiai, and the EEG showed temporal and parieto-occipital sharp waves or spikes. Headaches occurred in one child. The MRI showed no cortical structural involvement compatible with epileptogenic dysgenesis. An interictal SPECT showed cortical areas of hypoperfusion that correlated with the EEG focal abnormalities. A cerebellar hypoperfusion was also shown in 2 of the patients, suggesting an associated functional or structural lesion. (Iannetti P, Spalice A, Ciccoli C De F, et al. Seizures in paediatric Chiari type I malformation: the role of single-photon emission computed tomography. Acta Paediatr 2002;91:313-317). (Respond: P. Iannetti MD, Pediatric Neurology Department, La Sapienza University, Viale Regina Elena, 324, IT-00161 Roma, Italy).

COMMENT. Headache and neck pain are the most common symptoms associated with Chiari I malformation in childhood,. and seizures are infrequently reported. The MRI of the cerebrum is usually normal, and seizures are assumed to be cryptogenic. Brain SPECT, revealing a functional or structural lesion, appears to offer a cortical and/or cerebellar explanation for the seizures in some cases, when the MRI evidence for microdysgenesis is lacking. 